\title{
INJERTO DE MEMBRANA AMNIÓTICA CON ADHESIVO TISULAR EN EL TRATAMIENTO DE LA CONJUNTIVOCALASIA REFRACTIVA
}

\section{AMNIOTIC MEMBRANE TRANSPLANTATION WITH FIBRIN GLUE AS TREATMENT OF REFRACTORY CONJUNCTIVOCHALASIS}

\author{
FERNÁNDEZ-HORTELANO A ${ }^{1}$, MORENO-MONTAÑÉS J ${ }^{1}$, HERAS-MULERO H², \\ SADABA-ECHARRI LM ${ }^{1}$
}

\section{RESUMEN}

Caso clínico: Paciente de 63 años que presenta una conjuntivocalasia bilateral con lagrimeo, picor, escozor y retraso en el aclaramiento de fluoresceína. Al no observarse mejoría sintomática con el tratamiento tópico, se interviene quirúrgicamente con implante de membrana amniótica sin sutura.

Discusión: La conjuntivocalasia es una patología frecuente con síntomas comunes a la sequedad ocular. Cuando no hay respuesta al tratamiento médico se recurre al tratamiento quirúrgico. La técnica descrita por Tseng basada en el implante de membrana amniótica sin sutura resulta muy eficaz en estos casos debido a la poca inflamación que produce permitiendo una rápida recuperación y mejoría sintomática.

Palabras clave: Conjuntivocalasia, membrana amniótica, pegamento de fibrinógeno, sequedad ocular, inflamación.

\begin{abstract}
Case report: A 63-year-old man with bilateral conjunctivochalasis presented with tearing, irritation, foreign body sensation and a delayed fluorescein clearance test. After no symptomatic improvement with topical treatment, surgery was carried out, with amniotic membrane transplantation and fibrin sealant.

Discussion: Conjunctivochalasis is a frequent disorder that shares symptoms with dry eye syndrome. When there is no response to topical treatment, surgical treatment is needed. The surgical technique described by Tseng, and based on amniotic membrane transplantation without suture, resulted in a very useful response, due to less inflammation and a rapid resolution and improvement of symptoms (Arch Soc Esp Oftalmol 2007; 82: 571-574).
\end{abstract}

Key words: Conjunctivochalasis, amniotic membrane, fibrin glue, eye dryness, inflammation.

\footnotetext{
Recibido: 31/5/06. Aceptado: 25/7/07.

Departamento de Oftalmología. Clínica Universitaria de Navarra. Pamplona. España.

1 Doctor en Medicina.

2 Licenciado en Medicina.

Comunicación presentada en el LXXXII Congreso de la S.E.O. (A Coruña 2006).

Correspondencia:

A. Fernández Hortelano

Departamento de Oftalmología

Clínica Universitaria de Navarra

C/. Pío XII, s/n

31008 Pamplona

España

E-mail: anaferh@unav.es
} 


\section{INTRODUCCIÓN}

La conjuntivocalasia es un exceso de conjuntiva bulbar que se localiza principalmente entre el globo ocular y el párpado inferior. Tiende a ser bilateral, de localización inferior y es más frecuente en edad avanzada $(1,2)$. Las formas leves causan un agravamiento de la sequedad ocular preexistente con una película lagrimal inestable y retraso en el aclaramiento de fluoresceína. Las formas severas, sin embargo, pueden llevar a problemas oculares por exposición como úlceras corneales o dellen corneal.

Cuando la conjuntivocalasia es asintomática no es necesario tratarla. Si existen síntomas asociados, el tratamiento inicial consiste en lágrimas artificiales, oclusión ocular nocturna para disminuir la exposición nocturna y esteroides tópicos. Cuando los tratamientos tópicos fallan se recurre al tratamiento quirúrgico. Todos las técnicas quirúrgicas propuestas incluyen la excisión de la conjuntiva redundante. El método habitualmente descrito incluye una excisión simple de la conjuntiva inferior redundante y el cierre con suturas reabsorbibles (3).

La membrana amniótica se está utilizando con éxito para la reconstrucción de la superficie conjuntival y corneal. Debido a sus propiedades antiinflamatorias se ha utilizado con buenos resultados en la reconstrucción de la superficie conjuntival en la cirugía de la conjuntivocalasia (2).

\section{CASO CLÍNICO}

Varón de 63 años de edad que acude con síntomas de lagrimeo, conjuntivitis crónica y congestión nasal. A la exploración biomicroscópica se observa una conjuntivocalasia bilateral marcada (fig. 1) con un tiempo de aclaramiento de fluoresceína (FCT) que muestra una secreción basal elevada, un aclaramiento de fluoresceína enlentecido y una secreción lagrimal refleja normal.

Se instaura tratamiento con lágrimas artificiales sin conservantes, limpieza palpebral diaria y oclusión ocular sin objetivarse mejoría sintomática. Se añade al tratamiento colirio de metilprednisolona al $0,5 \%$ sin conservantes 3 veces al día durante 2 semanas. Se produce una mejoría sintomática transitoria, ya que los síntomas reaparecen al interrumpir el tratamiento.

Debido a la persistencia de estos síntomas, se sugiere el tratamiento quirúrgico bilateral, que se lleva a cabo según la técnica descrita por Tseng. Bajo anestesia tópica, se extirpa el exceso conjuntival. La esclera expuesta se recubre con membrana amniótica orientada con la membrana basal hacia arriba. Se pliega la membrana amniótica sobre sí misma para distribuir los dos componentes del pegamento de fibrinógeno (Tissucol ${ }^{\circledR}$ ) sobre la esclera y sobre la cara estromal de la membrana amniótica. El pegamento se distribuye en pequeñas gotas con ayuda de una cánula de cámara anterior. Se estira bien la membrana amniótica sobre la esclera para que se produzca una buena adhesión de ambos tejidos y se reseca el exceso de membrana amniótica. La conjuntiva residual se pega sobre la membrana. El tratamiento postoperatorio consiste en colirio metilprednisolona sin conservantes al $0,5 \% 3$ veces al día y colirio de ofloxacino 2 veces al día durante 15 días sin necesidad de llevar a cabo una oclusión postoperatoria. La mejoría biomicroscópica y sintomática se
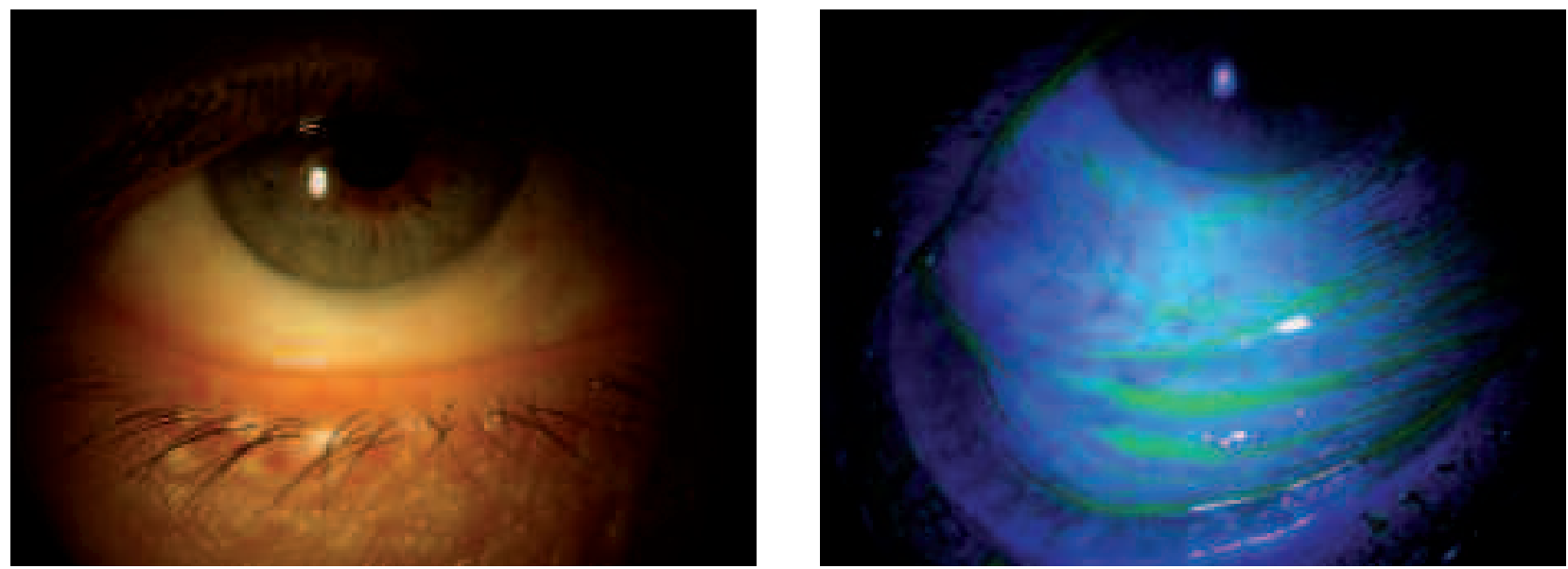

Fig. 1: Conjuntivocalasia antes de la cirugía. 
evidencia en el postoperatorio inmediato (fig. 2), manteniéndose en los meses de seguimiento (fig. 3).

\section{DISCUSIÓN}

La conjuntivocalasia es una conjuntiva redundante que se asocia a una pérdida parcial o completa de la cápsula de Tenon. La membrana amniótica puede ser un sustrato ideal para reforzar esta cápsula de Tenon gracias a sus propiedades. La membrana amniótica estimula la proliferación y diferenciación de las células epiteliales, mantiene el fenotipo epitelial original, disminuyendo la cicatrización, la vascularización y la inflamación.

El diagnóstico de la conjuntivocalasia a menudo se confunde con el de sequedad ocular pero la con- juntivocalasia no responde a tratamientos convencionales para el ojo seco. Es muy importante hacer un diagnóstico diferencial entre el ojo seco debido a un déficit de la secreción lagrimal acuosa y el ojo seco debido a la conjuntivocalasia (4).

Existen varias teorías sobre sobre el origen de la conjuntivocalasia. Una es la teoría mecánica, según la cual los cambios mecánicos asociados a la edad producen una obstrucción crónica del flujo linfático con la consiguiente dilatación linfática que lleva a la conjuntivocalasia clínica (5).

Otra de las principales teorías es la teoría inflamatoria, que se basa en una degradación excesiva de la matriz extracelular, con un tiempo de aclaramiento lagrimal retardado. Esto produce un acúmulo de enzimas de degradación en la lágrima con cambios inflamatorios crónicos que llevan a la laxi-
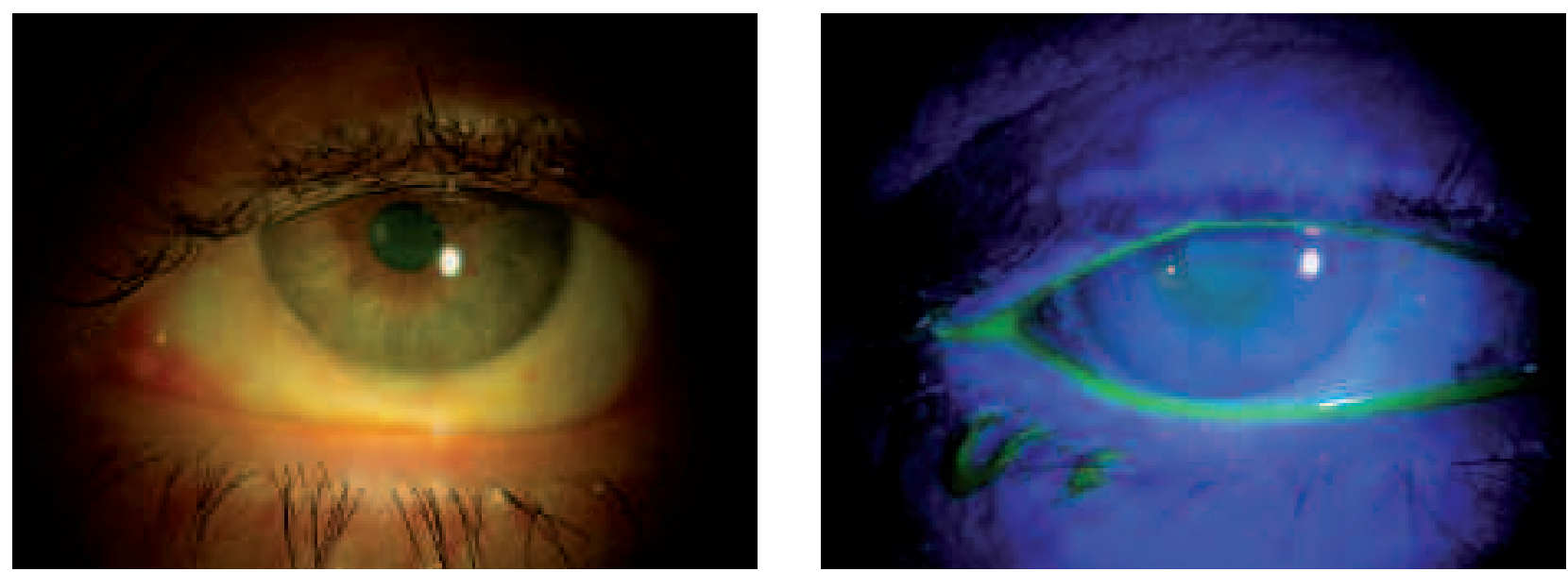

Fig. 2: Exploración biomicroscópica en el postoperatorio inmediato.
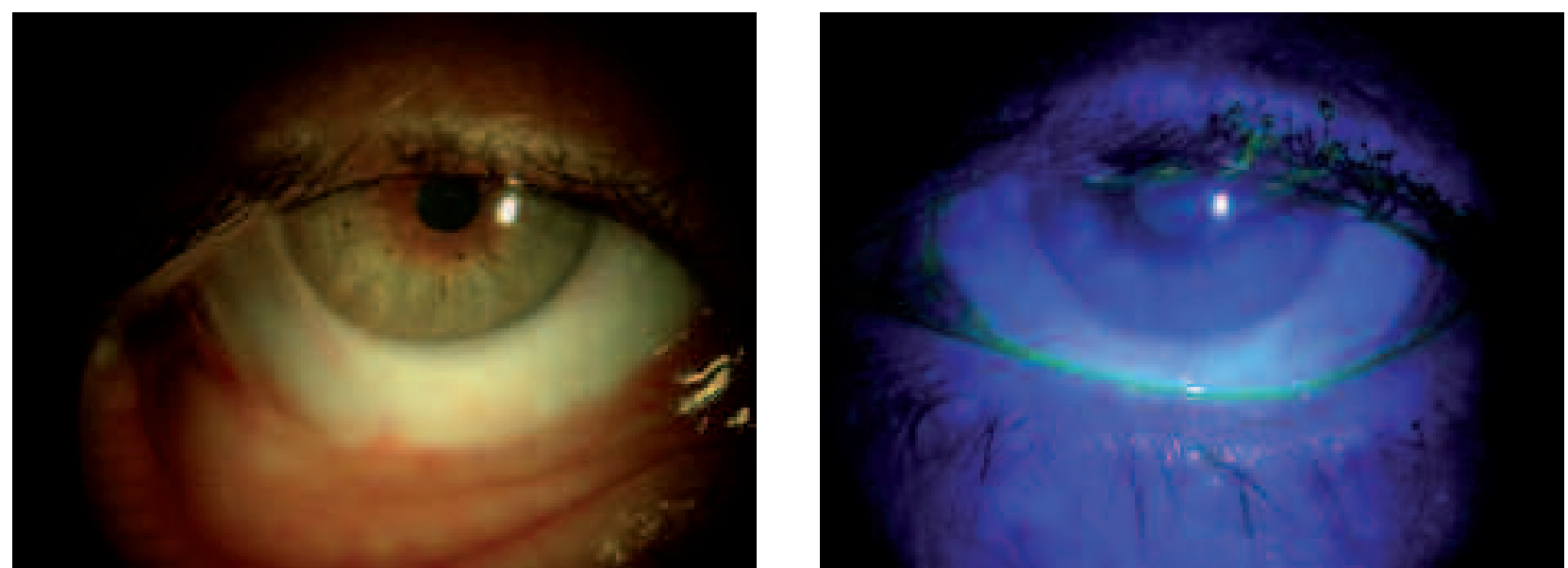

Fig. 3: Exploración biomicroscópica dos meses tras la cirugía. 
tud conjuntival o conjuntivocalasia (1). Apoyándonos en esta teoría, las propiedades antiinflamatorias de la membrana amniótica le permiten actuar directamente en el origen de la conjuntivocalasia.

Esta técnica quirúrgica produce una rápida recuperación debido a la menor inflamación postquirúrgica en comparación con la técnica con sutura. Además, mejora tanto los síntomas de conjuntivocalasia como los de la sequedad ocular asociada. Por tanto, consideramos que sería interesante llevar a cabo un estudio prospectivo aleatorizado que nos permita comparar la técnica descrita frente a la escisión simple y la escisión asociada a autoinjerto de conjuntiva en el tratamiento de la conjuntivocalasia refractaria a tratamiento médico convencional.

\section{BIBLIOGRAFÍA}

1. Meller D, Tseng SC. Conjunctivochalasis: literature review and possible pathophysiology. Surv Ophthalmol 1998; 43: 225-232.

2. Meller D, Maskin SL, Pires RT, Tseng SC. Amniotic membrane transplantation for symptomatic conjunctivochalasis refractory to medical treatments. Cornea 2000; 19: 796-803.

3. Hughes WL. Conjunctivochalasis. Am J Ophthalmol 1942; 25: 48-51.

4. Di Pascuale MA, Espana EM, Kawakita T, Tseng SC. Clinical characteristics of conjunctivochalasis with or without aqueous tear deficiency. Br J Ophthalmol 2004; 88: 388-392.

5. Watanabe A, Yokoi N, Kinoshita S, Hino Y, Tsuchihashi Y. Clinicopathologic study of conjunctivochalasis. Cornea 2004; 23: 294-298. 